\title{
Berichte aus der Zukunft? Ein methodologischer Essay zur Einleitung
}

Die Doppelsinnigkeit des Buchtitels ist bewusst gesetzt. Das „Umfeld“ von "Forschung" in einem weiteren empirischen Sinn wird in diesem Band anhand der „Feldforschung" untersucht. Oder auch: „Feldforschung“ wird in ihren Kontexten des gesamten Forschungsprozesses thematisiert.

Feldforschung in ihren engeren und weiteren Zusammenhängen also steht im Zentrum dieses Bandes. Ein Kunstgriff ist es nicht, mit dem die beiden Herausgeberinnen hier etwa versuchen würden, völlig disparate Themen mithilfe eines Wortspiels mühsam unter einen Hut zu bekommen. Es verhält sich geradezu umgekehrt: „Feldforschung“ galt einst als problemloser, eindeutiger und klarer Standardbegriff, der sich quer durch die Wissenschaftsgeschichte des 20. Jahrhunderts aber so weit differenziert und subtil weiter entwickelt hat, dass darauf heute kaum treffender als mit einem mehrdeutigen Titel zu verweisen wäre.

Die scheinbar einheitlichen, klaren und überschaubaren Anfänge der wissenschaftlichen Feldforschung wurzeln im 19. Jahrhundert, als eine explizite Nähe und symmetrische Parallelität zwischen "Geistes- und Naturwissenschaften“ im deutschsprachigen Raum dem Humboldt'schen Ideal von der Gesamt-Universität zu folgen suchte. Bei allen Unterschieden zu den französisch- und englischsprachigen Wissenschaftslandschaften, mit ihren von Anfang an stärkeren Prioritäten für die Naturwissenschaften, lassen sich ähnliche Anfänge auch dort ausloten. Die damaligen Konzepte von „field research“ und „enquête du terrain“ machen sogar noch deutlicher als ihr deutschsprachiges Gegenstück, wo die akademischen Anfänge und Auslöser von „Feldforschungen“ lagen, und von „Feldstudien“ als ihren bescheideneren Versionen. Es waren der „Feldversuch“" und letztlich das „Experiment“, die innerhalb des historischen Wissenschaftsbetriebes das semantische und logistische Vorbild abgaben für das, was sich in Anlehnung daran allmählich in einem Teil der biologischen Fächer und der Geisteswissenschaften als „Feldforschung“ zu entwickeln begann. 
Mit Unterscheidungen wie jenen zwischen nomothetischen und idiographischen Zugängen hatten Versuche eingesetzt, die differenten Zugänge zur jeweiligen Erkenntnis besser abzuschätzen. Stärker als andere, und frühzeitiger, verabsolutierte aber gerade die deutschsprachige Tradition die entsprechende Verschiedenartigkeit zwischen „Natur- und Geisteswissenschaften“, bis hinein in die institutionellen, organisatorischen und diskursiven Feinheiten des akademischen Lebens. Demnach benötigte auch das Ideal der "Geisteswissenschaften" seine eigenen Kriterien der empirischen Erkenntnis, als gültige und plausible Gütesiegel der Qualität von wissenschaftlicher Wahrheit. Parallel zur abstrakten und logischen Ebene der theoretischen und methodologischen Voraussetzungen und Rahmenbedingungen wurde ein anderes dieser Gütesiegel auf der Ebene der dokumentierten Empirie identifiziert. In ihr waren jene Daten zu erheben, welche Interpretationen, Modelle und Theorien speisten; an ihr waren jene Interpretationen, Modelle und Theorien zu prüfen und zu erproben.

Was das Experiment und der Feldversuch für Physik, Chemie oder Medizin waren, das also wurden Feldstudie und Feldforschung für Teile von Zoologie und physischer Anthropologie, von Völkerkunde und Musikwissenschaften, von Linguistik und regionalen Philologien. Und so wie exakte Messinstrumente und kontrollierte Rahmenbedingungen als notwendig erkannt wurden zur korrekten Durchführung und Auswertung von Experiment und Feldversuch, so wurden verlässliche Gerätschaften geradezu eine conditio sine qua non für gute Feldstudien und Feldforschungen. Aus derartigen historischen Kontexten und Leitorientierungen heraus entwickelte sich in meinem eigenen Fach in jenen Jahrzehnten als eine besonders markante und spezialisiertere Version des Ganzen die ethnologische Feldforschung - mit Wurzeln, die über Heinrich Barth, Alois Musil, Franz Boas und Bronislaw Malinowski deutlich nach Mitteleuropa verweisen. Aus eben diesen weiteren regionalen und wissenschaftsgeschichtlichen Kontexten heraus wurde auch das Phonogrammarchiv der Österreichischen Akademie der Wissenschaften gegen Ende des 19. Jahrhunderts gegründet und aufgebaut, dem die Herausgeberinnen angehören, dessen Kuratoriums-Obmann ich von 2003 bis 2007 war, und das jene Vortragsreihe veranstaltete, aus der dieses Buch hervorgeht.

Was gegen Ende des 19. Jahrhunderts vielleicht selbstverständlicher war, ist heute eine Ausnahme. Heutzutage ist es eine Seltenheit geworden, dass sich Vertreter/innen aus Musikwissenschaften, Linguistik, Zoologie, Politikwissenschaft, Afrikanistik, Turkologie, Philologien, Soziologie, Theaterwissenschaft, Europäischer Ethnologie sowie aus Kultur- und Sozialanthropologie zu einem gemeinsamen methodischen Thema in ein und demselben Sammelband zu Wort melden. Dass aus unterschiedlichsten Disziplinen zu diesem oder jenem übergeordneten Thema etwas beigesteuert wird, das kommt alle paar Wochen vor, das ist normaler 
akademischer Alltag und wäre nicht weiter Aufsehen erregend. Wenn es sich aber um verschiedenste Fachbeiträge zu einem methodischen Thema handelt, dann kann ohne Zögern festgestellt werden: Zu Beginn des 21. Jahrhunderts kommt so etwas höchstens alle paar Jahre vor, egal ob in deutscher oder in einer anderen Sprache. Steckt mehr hinter diesem Publikationsereignis der seltenen Art, als dass hier eben Beiträge versammelt sind aufgrund der Initiative von zwei kreativen Vertreterinnen einer Einrichtung, die sich seit über hundert Jahren der Dokumentation empirischer Forschung verpflichtet hat? Schon die Initiative ist einer Würdigung wert. Es stellt eine besondere, und eine besonders wertvolle Anstrengung dar, die Vertreter/innen dieser unterschiedlichen Fachgebiete dafür zu gewinnen, eine gemeinsame und übergreifende methodische Fragestellung zu reflektieren, welche die methodischen und empirischen Grundlagen des eigenen wissenschaftlichen Arbeitens schlechthin betrifft. Bemerkenswert sind aber vor allem die Ergebnisse dieser Initiative. Sie zeigen Neues an, worüber nachzudenken sich lohnt.

Vielleicht zeigen die vorliegenden Ergebnisse dieses Bandes neben allem Reichtum im Detail um ein weiteres Mal an, dass wir uns dem Ende einer älteren Ära der Wissenschaftsgeschichte nähern, und dass die Elemente einer neuen Ära heranreifen.

Die noch anhaltende, ältere Ära der Wissenschaftsgeschichte war seit dem Ende des zweiten Weltkriegs geprägt vom Schlagwort der „,zwei Kulturen“: „Naturwissenschaften “ machen das Eine, „Geistes- und Sozialwissenschaften“ machen das Andere. Die Angehörigen dieser beiden akademischen Großgemeinschaften verstünden sich selbst im Prinzip eher schon, aber die Angehörigen der anderen Großgemeinschaft jeweils überhaupt nicht. Nicht nur Erkenntnisformen und Wahrheitskriterien, sondern auch Arbeitsweisen, Kommunikationsmodi und Lebensstile seien nach außen hin derart verschieden, dass tatsächlich von zwei unterschiedlichen Wissenschaftskulturen zu sprechen sei. Im Inneren dieser beiden Großgemeinschaften würde dies durch zunehmende Arbeitsteilung auf ganz ähnliche Weise dupliziert und repliziert. Das Schlagwort von den „zwei Kulturen“ charakterisierte tatsächlich bis zu einem gewissen Grad seit 1945 über Jahrzehnte charakteristische Aspekte der Realitäten. Dennoch enthielten sowohl das Schlagwort als auch die ihm entsprechenden Realitäten eine geradezu unglaubliche Paradoxie.

Der Nationalsozialismus war eben erst nieder gerungen worden. Zugleich wurde auf diesem Weg eine Sicht und Praxis des Wissenschaftsbetriebes weltweit verallgemeinert, die bis dahin in dieser Absolutheit ein spezifisch-deutschsprachiges Erbe des Wissenschaftsbetriebes gewesen waren. Die Setzung von absoluter Verschiedenheit zwischen „Natur- und Geisteswissenschaften“ war ein globales Novum, das in dieser Form wohl nur als Antithese zur politischen Instrumentalisierung der 
Wissenschaften durch totalitäre Regimes zu interpretieren ist: Der liberale Staat beanspruchte eben nicht für eigene Zwecke zu instrumentalisieren, und obwohl er Wissenschaften sponserte, ließ er ihre „eigenen Kulturen“ zu. Die Ära der „zwei Kulturen" war und ist eine Zeit der paradoxen Getrenntheit. Meiner Ansicht und Hoffnung nach geht diese Ära ihrem Ende entgegen. Die paradoxe Getrenntheit wird von beiden Seiten her zunehmend ausgehöhlt, hinterfragt, durchbrochen. In die eine Richtung hin war einer unter vielen Indikatoren dafür der "Science War“ der anglophonen akademischen Welt in den 1990er Jahren, mit einem für manche peinlichen Auslöser: Ein geisteswissenschaftliches Magazin, das sich der subjektiven Interpretation und der Postmoderne verschrieben hatte, publizierte einen bewusst konstruierten naturwissenschaftlichen Humbug, der sich als kritisch und antipositivistisch getarnt hatte. Ganz andere Indikatoren für die schrittweise Auflösung der „Zwei Kulturen“ sehe ich aber auch in die umgekehrte Richtung wirken: dort nämlich, wo seriöse Naturwissenschafter wie etwa Anton Zeilinger darüber nachdenken, inwiefern die heutigen Naturwissenschaften nicht in einer Logik der Ordnung verfangen sein könnten, die durch die Grenzen der europäischen Kulturgeschichte gesetzt sind. Wenn Naturwissenschafter/innen also zu überlegen beginnen, ob ihre eigenen Prämissen nicht euro-zentrisch sind und etwa durch die Axiome asiatischer Philosophien besser zu überschreiten wären, lösen sich die Grenzen der „zwei Kulturen“ auf.

Offensichtlich haben „beide Kulturen“ begonnen, sich ihrer eigenen Beschränktheiten gewahr zu werden, und diese aufzuweichen und $\mathrm{zu}$ transformieren. Ich halte diese Transformation der alten „zwei Kulturen“ für eine gute Entwicklung, obwohl ich weiß, dass mächtige Kräfte versuchen, diese Entwicklung für sich zu nutzen, um Budgets einzusparen und Forschungsmöglichkeiten einzuengen. Als genereller Tendenz ist diesem Ansinnen standzuhalten. Solches aber wäre unmöglich mit einer konservativen Orientierung zur Aufrechterhaltung und Verteidigung der alten Welt von „zwei Kulturen“. Das erschöpfte, konservative Verteidigen einer untergehenden Wissenschaftswelt der Nachkriegszeit nach 1945 hat in dieser Form keine Zukunft. In meinem Verständnis kommt die Ära nach der Zeit der „zwei Kulturen" mit Sicherheit, aus innerwissenschaftlichen Gründen. Die entscheidende Frage wird daher nicht sein, ob sie kommt, sondern wer sie wie gestaltet, innerhalb und außerhalb der Wissenschaften.

Das ist der Hauptgrund, warum ich den vorliegenden Band so interessant und zukunftsweisend finde. Das monolithische Wissenschaftsbild des 19. Jahrhunderts ist obsolet, und die Zeit totalitärer Instrumentalisierungen der Wissenschaften ist ebenso vorbei. Die Zeit der „zwei Kulturen“ geht ihrem Ende schneller entgegen als vielen lieb ist. Wem daher die Zukunft guter und kritischer wissenschaftlicher Arbeiten ein Anliegen ist, der engagiert sich auch und vor allem für ihre sachlichen 
Prinzipien, ihre methodischen Verfahren, ihre empirischen Grundlagen, ihre diskursiven Kommunikationsformen und ihre interpretativen Spielräume. Wir sind zum einen bescheidener und selbstkritischer geworden in unserem wissenschaftlichen Selbstverständnis. Das bedeutet aber zugleich, dass wir gelernt haben, sorgfältiger, flexibler und genauer zuzuhören und zuzusehen. Selbstreflexive neue Formen von Realismus werden in vielen Beiträgen dieses Bandes angezeigt. Anhand dieses Bandes zeigt sich: Neue Clusters, Bündel und Zentren der Forschungspraxis bilden sich heraus, quer zu den Grenzen der alten „zwei Kulturen“. Ähnlich wie die „zwei Blöcke“ der politischen Welt nach 1945 untergegangen sind, verschwinden mit Zeitverzögerung auch die „zwei Kulturen“ der akademischen Welt nach 1945. An ihrer Stelle wird eine neue Galaxis von beweglichen, großen und kleinen Konstellationen sichtbar, denen in vielen neuen Formen die Feldforschung wesentlich bleibt. 
